\title{
Rethink Your Transjugular Intrahepatic Portosystemic Shunt (TIPS): Pre-TIPS Infection Predicts Post-TIPS Infection and Post-TIPS Portosystemic Encephalopathy
}

\author{
Authors: \\ Jenna E. Koblinski, ${ }^{1}$ Margaret C. Liu,, Roy U. Bisht,, ${ }^{1}$ Paul Kang, ${ }^{2}$ Mark N. \\ Wong, $3,4 *$ Ester C. Little ${ }^{3,4}$ \\ 1. University of Arizona College of Medicine - Phoenix, Phoenix, Arizona, USA \\ 2. Mel and Enid Zuckerman College of Public Health, Tucson, Arizona, USA \\ 3. Advanced Liver Disease and Transplant Institute, Banner - University Medical \\ Center Phoenix, Phoenix, Arizona, USA \\ 4. Department of Internal Medicine, University of Arizona College of Medicine, \\ Phoenix, Arizona, USA \\ *Correspondence to Ester.Little@bannerhealth.com \\ Disclosure: $\quad$ Ester Little has received research grants from Intercept Pharmaceuticals. Other \\ authors have not declared any conflicts of interests. \\ Acknowledgements: The authors are forever thankful to the Interventional Radiology team at \\ Banner - University Medical Center Phoenix who performed the TIPS procedure \\ during the years of the study: Dr Kevin Hirsh, Dr David Wood, Dr Steven Chen, and \\ Dr Cole Mendenhall. \\ Received: \\ 16.12 .19 \\ Accepted: \\ 23.03.20 \\ Keywords: \\ Ascites, cirrhosis, infection, portal hypertension, portal systemic encephalopathy, \\ transjugular intrahepatic portosystemic shunt (TIPS). \\ Citation: \\ EMJ Hepatol. 2020;8[1]:34-41.
}

\section{Abstract}

Objective: Transjugular intrahepatic portosystemic shunt (TIPS) is used for decompression of elevated portal pressure; however, there are potential complications. The aim of this study was to compare the risk of complications of TIPS in those who had an episode of infection within 6 months prior to TIPS to those without an infection prior.

Methods: A retrospective chart review was performed on patients who underwent TIPS at a single transplant centre over 8 years. They were divided into two groups: patients without infection during the 6 months prior to TIPS ( $n=349)$ and those with an infection prior (bacterial/fungal) $(n=53)$. The Wilcoxon rank-sum test was used to compare continuous variables while chi-squared analysis and Fisher's exact test was used for categorical variables. Multiple logistic regression was used to ascertain the association between pre-TIPS infection status and likelihood of post-TIPS infection.

Results: In the group of patients who had an infection before TIPS, $26.4 \%(n=14)$ had an episode of infection after the procedure, while in the group without infection prior, $16.2 \%(n=55)$ had an infection after the procedure ( $p=0.047$; odds ratio: 2.08 ). In the pre-TIPS infection group, 54.7\% $(n=29)$ had an episode of portosystemic encephalopathy post-TIPS versus 39.6\% $(n=134)$ in the group without infection before TIPS ( $p=0.046$; odds ratio: 1.93). 
Conclusion: Pre-TIPS infection within 6 months of TIPS procedure is a risk factor for post-TIPS portosystemic encephalopathy and infection. Further studies are needed to determine the potential benefit of antibiotic prophylaxis in patients who had an infection in the 6 months preceding TIPS placement.

\section{INTRODUCTION}

Transjugular intrahepatic portosystemic shunt (TIPS) is widely used for the decompression of elevated portal pressure. Several studies have demonstrated that TIPS is very effective for secondary prevention of oesophageal variceal bleeding and treatment of refractory ascites, Budd-Chiari syndrome, hepatic hydrothorax, ectopic varices, and more recently, portal vein thrombosis. ${ }^{1-4}$

Complications of the TIPS procedure include portosystemic encephalopathy (PSE), infection, bleeding, respiratory complications, and liver failure. ${ }^{5-7}$ The model of end stage liver disease (MELD), and later its modification to include serum sodium in the calculation (MELD-Na), has been proven to be the best model to predict mortality after TIPS placement., 3,-13

Other factors have been identified as predictors for specific complications of TIPS. Increased age, prior episodes of PSE, and a higher Child-Pugh class have been identified as predictors of postTIPS portosystemic hepatic encephalopathy. ${ }^{14}$ TIPS inserted for control of acute variceal bleeding and use of overlapping shunts at stent insertion have been shown to be risk factors for infection of the TIPS shunt ('tipsitis') and for postTIPS unexplained sustained bacteraemia. ${ }^{15}$

Patients with cirrhosis have an increased risk of infections in general including infection of the ascitic fluid, particularly spontaneous bacterial peritonitis. ${ }^{16-20}$ Similar to the known risk factors that predict certain complications of TIPS, there are known predisposing factors that can predict the probability of spontaneous bacterial peritonitis in patients with ascites, such as low serum sodium levels, Child-Pugh Stage C, and elevated ascitic polymorphonuclear cell counts. ${ }^{21}$ However, there are insufficient data about whether an infection (bacterial or fungal) prior to the insertion of the TIPS can affect the rate of complications after the procedure. The aim of the study was to compare the rate of complications of TIPS in patients who

had an episode of infection within 6 months prior to TIPS and those without a history of infection preceding the procedure.

\section{METHODS}

A retrospective chart review was performed on all patients who underwent TIPS procedure at the Banner - University Medical Center Phoenix between January 2010 and April 2018. The study was approved by the local Institutional Review Board (IRB). All patients had a polytetrafluoroethylene covered Viatorr $^{\odot}$ stent (WL Gore, Flagstaff, Arizona, USA). Patients' clinical characteristics, including demographics, laboratory tests before and after the procedure, cause of portal hypertension, and the indication for TIPS placement, were collected. Data on episodes of infection (bacterial or fungal) in the 6 months prior to TIPS, complications of the procedure, and outcomes post-TIPS were also collected. The information was derived from the patients' electronic medical records. The laboratory tests conducted closest to the day of the procedure were utilised for the tables and the MELD-Na score calculations.

Prior to the TIPS procedure, and in addition to routine clinical history, physical exam, and laboratory tests, all patients had an ECG to make sure the ejection fraction was normal. In addition, all patients had a CT or MRI to evaluate the anatomy of the liver and its vascular structures, as well as to exclude the possibility of liver cancer. Upon discharge after TIPS insertion, the patients were instructed to return to the radiology department for a Doppler ultrasound to check for TIPS patency 1-2 weeks after discharge. If any possible complications of the procedure were identified at that point, the patients were either admitted to the hospital or referred to the hepatology clinic for an appointment.

During the study period, there were 402 patients who underwent TIPS and were divided into two groups: those who had no infection during the 6 months prior to TIPS $(n=349)$, and those 
who did have an episode of infection (bacterial or fungal) during the 6 months preceding the procedure $(n=53)$.

The diagnosis of infection, both prior to and after TIPS, was based on clinical data, laboratory tests, and imaging results that when combined prompted the admitting doctors to start antibiotic therapy. Patient demographic and baseline clinical characteristics in those with and without infection 6 months prior to the TIPS procedure were reported as means, standard deviations for continuous variables, and frequencies and percentages for categorical variables. The 6-month period was chosen arbitrarily as a period that would capture infections that could possibly interfere with the outcome of the TIPS procedure. The Wilcoxon rank-sum test was used to compare continuous variables between the groups, while chi-squared analysis and Fisher's exact test were used to compare the categorical variables. Logistic regression was implemented to ascertain the odds of clinical outcomes relative to pre-TIPS infection status with patients with no infection as a reference group. All models were adjusted for age, sex, race, pre-TIPS and post-TIPS lab values, dialysis, and pre-TIPS PSE statuses. All $\mathrm{p}$ values were two-sided and $\mathrm{p}<0.05$ was considered statistically significant. All analyses were conducted using STATA version $14^{\circ}$ (STATA Corp; College Station, Texas, USA).

\section{RESULTS}

The group of patients that had an infection during the 6 months prior to TIPS was referred to as Group A, and the group of patients that did not have an infection during the 6 months prior to TIPS was referred to as Group B.

The two groups were similar in the distribution of age, sex, and ethnicity. The mean age in years for Group A was 56.9 and for Group B was 57.7 $(p=0.67)$. Group A consisted of $60.4 \%$ males and Group B was $61.4 \%$ males ( $p=0.88$ ). In Group A, $84.3 \%$ of the patients were Caucasian (27.0\% Hispanic), $3.9 \%$ were African American, and $11.8 \%$ were American Indian or Alaska Native. In Group B, 88.1\% were Caucasian (27.7\% Hispanic), $0.6 \%$ were African American, 3.0\% were Asian American, and $10.3 \%$ were American Indian or Alaska Native. There was no statistically significant difference between ethnicity or race between the two groups $(p=0.2)$.
The aetiology of liver disease was also very similar in the two groups. Hepatitis C, alcoholic liver disease, and nonalcoholic fatty liver disease were the most common aetiologies of portal hypertension in both groups. In Group A, the aetiology of liver disease was hepatitis C in 33.3\% of the patients, $31.9 \%$ had alcoholic liver disease, 20.3\% had nonalcoholic fatty liver disease, $4.4 \%$ had autoimmune hepatitis, $2.9 \%$ had primary biliary cholangitis, $1.5 \%$ had haemochromatosis, and $13.0 \%$ had other causes of liver disease, with some patients having more than one aetiology. The aetiology of the liver disease in Group B was $31.6 \%$ hepatitis C, 39.9\% alcoholic liver disease, $19.2 \%$ nonalcoholic fatty liver disease, $3.1 \%$ autoimmune hepatitis, $2.5 \%$ primary biliary cholangitis, $1.2 \%$ haemochromatosis, $0.6 \%$ a-1antitrypsin deficiency, $0.6 \%$ hepatitis B, $0.3 \%$ hepatocellular carcinoma, and $13.6 \%$ had other causes of liver disease. Some patients also had more than one aetiology. The only statistically significant difference in aetiology between the two groups was for alcoholic liver disease, which was more common in Group B, patients who did not have an infection in the 6 months prior to $\operatorname{TIPS}(p=0.019)$.

The indications for TIPS placement were also comparable between the two groups (Table 1), except for patients requiring TIPS for both ascites and hepatic hydrothorax, which was more frequently seen in patients in Group A (11.3\% of the patients in Group $A$ and $4.5 \%$ of patients in Group B [p=0.045]).

Table 2 shows the laboratory tests, results, and the MELD-Na of the two groups before and after TIPS. Prior to TIPS, the patients in Group A had a statistically significant higher MELD-Na score, with a lower serum sodium and higher international normalised ratio, creatinine, and total bilirubin. However, the difference in serum albumin and the percentage of patients who required haemodialysis were not significantly different amongst the two groups. In the post-TIPS laboratory tests, the MELD-Na and creatinine remained higher in Group A. The serum sodium, total bilirubin, and albumin were different between the groups, but the differences were not statistically significant. However, the percentage of patients requiring dialysis post-TIPS was significantly higher in Group A. 
Table 3 depicts the rate of complications of TIPS in the two groups. Before TIPS, PSE was seen in $52.8 \%$ of the patients in Group A and in $32.1 \%$ of the patients in Group B. After TIPS, 54.7\% $(n=29)$ of patients in Group A and 39.6\% ( $n=134)$ of patients in Group B had PSE ( $p=0.046$; odds ratio [OR]: 1.93). Of the patients in Group A, 26.4\% ( $n=14)$ had an infection post-TIPS, whereas $16.2 \%(n=55)$ of patients in Group B had an infection post-TIPS ( $p=0.047 ;$ OR: 2.08). Except for PSE and infection, there were no statistically significant differences between the two groups for other complications of TIPS including hospitalisation within 90 days, acute kidney injury requiring haemodialysis, procedural haemorrhage, gastrointestinal haemorrhage, respiratory complications, or death.

\section{DISCUSSION}

The TIPS procedure is the leading portosystemic shunt performed in the USA. With 4.5 million adults in the USA diagnosed with liver disease, many of whom will develop cirrhosis and its complications, it is crucial to better delineate the complications of the procedure and potential risk factors associated with those complications. ${ }^{22,23}$ While there are known risk factors for some specific complications of TIPS, there are limited data about if and how an infection pre-TIPS affects patients post-TIPS. This is an important factor to consider because infection, whether bacterial or fungal, is a potentially modifiable risk factor.

Prior to the data collection, the authors hypothesised that an episode of infection (fungal or bacterial) pre-TIPS placement would cause an increase in the number of infections after the procedure, and that an episode of infection prior to the procedure had the potential to increase the rate of other complications of TIPS. These results confirmed the hypothesis that infection pre-TIPS increased the risk of infection post-TIPS, with post-TIPS infection seen in $26 \%$ of the patients in Group A, compared with $16 \%$ of the patients in Group B. This difference was statistically significant. Logistic regression was used to ascertain the odds of clinical outcomes relative to pre-TIPS infection status, with patients with no infection as the reference group.

Table 1: Indications for TIPS in patients with versus those without infection in the 6 months prior to TIPS placement.

\begin{tabular}{|c|c|c|c|}
\hline Variables & $\begin{array}{c}\text { Group A } \\
n=66\end{array}$ & $\begin{array}{c}\text { Group B } \\
n=387\end{array}$ & $\mathrm{p}$ value \\
\hline \multicolumn{4}{|l|}{$\begin{array}{c}\text { Indications } \\
\mathrm{n}(\%)\end{array}$} \\
\hline Acute variceal bleeding & $22(42.0)$ & $123(35.0)$ & 0.37 \\
\hline Ascites & $21(40.0)$ & $122(35.0)$ & 0.5 \\
\hline Hydrothorax & $3(6.0)$ & $15(4.0)$ & 0.71 \\
\hline Ascites and hydrothorax & $6(11.0)$ & $16(5.0)$ & 0.045 \\
\hline $\begin{array}{c}\text { Oesophageal variceal } \\
\text { bleeding not responsive to } \\
\text { banding }\end{array}$ & $2(3.0)$ & $15(4.0)$ & 1 \\
\hline Non-bleeding gastric varix & $4(8.0)$ & $25(7.0)$ & 1 \\
\hline Portal vein thrombus & $7(13.0)$ & $64(18.0)$ & 0.36 \\
\hline Budd-Chiari & $1(2.0)$ & $6(2.0)$ & 1 \\
\hline Hepatorenal & $0(0.0)$ & $1(0.3)$ & 1 \\
\hline
\end{tabular}

$\mathrm{p}$ values were calculated using the Wilcoxon rank-sum test to compare continuous variables. Chi-squared and Fisher's exact were used to compare categorical variables. The number of patients listed in the table is greater than the total number of patients enrolled in the study because patients may have had multiple indications for TIPS. Group A: patients that had an infection during the 6 months prior to TIPS.

Group B: patients that did not have an infection during the 6 months prior to TIPS.

TIPS: transjugular intrahepatic portosystemic shunt. 
Table 2: Laboratory values pre-TIPS and post-TIPS in patients with versus those without infection in the 6 months prior to TIPS placement.

\begin{tabular}{|c|c|c|c|}
\hline Variables & $\begin{array}{c}\text { Group A } \\
n=53\end{array}$ & $\begin{array}{l}\text { Group B } \\
n=349\end{array}$ & $\mathrm{p}$ value \\
\hline \multicolumn{4}{|l|}{ Pre-TIPS } \\
\hline Haemoglobin (mean, SD) & $9.33(+/-2.31)$ & $10.00(+/-2.39)$ & 0.03 \\
\hline INR (mean, SD) & $1.87(+/-0.83)$ & $1.57(+/-0.81)$ & $<0.001$ \\
\hline Dialysis (yes, \%) & $4(+/-7.55)$ & $11(+/-3.20)$ & 0.12 \\
\hline Creatinine (mean, SD) & $1.44(+/-0.89)$ & $1.19(+/-0.98)$ & 0.003 \\
\hline Albumin (mean, SD) & $2.65(+/-0.74)$ & $2.73(+/-0.72)$ & 0.44 \\
\hline Total bilirubin (mean, SD) & $4.03(+/-6.29)$ & $2.56(+/-3.55)$ & 0.006 \\
\hline Sodium (mean, SD) & $135.8(+/-5.63)$ & $137.7(+/-5.53)$ & 0.026 \\
\hline MELD-Na (mean, SD) & $21.8(+/-9.70)$ & $16.8(+/-6.99)$ & $<0.001$ \\
\hline \multicolumn{4}{|l|}{ Post-TIPS } \\
\hline Haemoglobin (mean, SD) & $9.25(+/-1.70)$ & $9.85(+/-1.97)$ & 0.02 \\
\hline INR (mean, SD) & $1.96(+/-0.70)$ & $1.71(+/-0.59)$ & 0.004 \\
\hline Dialysis (yes, \%) & $6(+/-11.80)$ & $11(+/-3.24)$ & 0.015 \\
\hline Creatinine (mean, SD) & $1.29(+/-0.75)$ & $1.12(+/-1.00)$ & 0.005 \\
\hline Albumin (mean, SD) & $2.49(+/-0.61)$ & $2.60(+/-0.66)$ & 0.18 \\
\hline Total bilirubin (mean, SD) & $4.69(+/-5.56)$ & $3.75(+/-4.66)$ & 0.27 \\
\hline Sodium (mean, SD) & $138.2(+/-5.46)$ & $139.3(+/-5.57)$ & 0.21 \\
\hline MELD-Na (mean, SD) & $22.4(+/-8.80)$ & $18.7(+/-6.81)$ & 0.006 \\
\hline
\end{tabular}

$\mathrm{p}$ values were calculated using the Wilcoxon rank-sum test to compare continuous variables. Chi-squared and Fisher's exact were used to compare categorical variables.

Group A: patients that had an infection during the 6 months prior to TIPS.

Group B: patients that did not have an infection during the 6 months prior to TIPS.

INR: international normalised ratio; MELD-Na: model of end stage liver disease-sodium; SD: standard deviation; TIPS: transjugular intrahepatic portosystemic shunt.

An episode of infection within 6 months of TIPS placement was a predictor of post-procedure infection ( $p=0.047$; OR: 2.08).

In 2016, Deng et al. ${ }^{24}$ looked at risk factors associated with early infection following TIPS procedure. The authors identified cholangiolithiasis, Child-Pugh Class C, and hepatitis $C$ virus infection to be correlated with fever post-TIPS. This finding was statistically significant. There was no correlation between infection and factors such as age, sex, and diabetes. Episodes of infection before TIPS as possible predictors of post-TIPS infection were not included. ${ }^{24}$
In a more recent publication, Vozzo et al. ${ }^{25}$ looked at 30-day readmission after TIPS placement and found that $36 \%$ of the patients were readmitted to the hospital. In their study, the authors identified the most common reasons for admission as hepatic encephalopathy (48\%), infection (15\%), bleeding (11\%), and fluid overload (7\%). The percentage of patients who were readmitted because of infection were similar to that of the patients in this present study who had no episodes of infection in the 6 months prior to TIPS placement. However, in the study by Vozzo et al., ${ }^{25}$ the authors did not comment on whether or not the patients readmitted because of infection had previously had an episode of infection prior to TIPS placement. 
Table 3: Complication of TIPS procedure in patients with versus those without infection in the 6 months prior to TIPS placement.

\begin{tabular}{|c|c|c|c|c|}
\hline Outcomes & $\begin{array}{l}\text { Group A } \\
n=53\end{array}$ & $\begin{array}{l}\text { Group B } \\
n=349\end{array}$ & OR $(95 \% \mathrm{Cl})$ & $\mathrm{p}$ value \\
\hline $\begin{array}{l}\text { Hospitalisation within } \\
90 \text { days (yes, \%) }\end{array}$ & $31(62.0)$ & $181(53.4)$ & $1.43(0.74-2.99)$ & 0.25 \\
\hline PSE (yes, \%) & $29(54.7)$ & $134(39.6)$ & $1.93(1.01-3.70)$ & 0.046 \\
\hline $\begin{array}{c}\text { Post-TIPS infection } \\
\text { (yes, \%) }\end{array}$ & $14(26.4)$ & $55(16.2)$ & $2.08(1.01-4.29)$ & 0.047 \\
\hline AKI Req HD (yes, \%) & $5(9.4)$ & $27(8.0)$ & $0.17(0.02-1.38)$ & 0.09 \\
\hline $\begin{array}{c}\text { Procedural } \\
\text { haemorrhage (yes, } \\
\%)\end{array}$ & $0(0.0)$ & $13(3.9)$ & N/A & \\
\hline $\begin{array}{c}\text { Gastrointestinal } \\
\text { haemorrhage (yes, } \\
\% \text { ) }\end{array}$ & $8(15.1)$ & $33(9.8)$ & $2.32(0.71-7.55)$ & 0.15 \\
\hline $\begin{array}{c}\text { Respiratory } \\
\text { complication (yes, \%) }\end{array}$ & $11(20.8)$ & $67(22.6)$ & $0.26(0.05-1.32)$ & 0.1 \\
\hline Other (yes, \%) & $6(11.5)$ & $58(17.2)$ & $0.79(0.22-2.81)$ & 0.71 \\
\hline Death (yes, \%) & $17(32.1)$ & $82(24.2)$ & $1.08(0.51-2.31)$ & 0.83 \\
\hline
\end{tabular}

Odds ratios and 95\% confidence intervals were calculated using multiple logistic regression adjusting for pre-TIPS and post-TIPS lab values, dialysis, and PSE Pre-TIPS.

Group A: patients that had an infection during the 6 months prior to TIPS.

Group B: patients that did not have an infection during the 6 months prior to TIPS.

AKI Req HD: acute kidney injury requiring haemodialysis; PSE: portosystemic encephalopathy; TIPS: transjugular intrahepatic portosystemic shunt.

In a study from Allaire et al. ${ }^{26}$ presented at the European Association for the Study of Liver (EASL) meeting in 2019, the authors reported the survival and risk factors of mortality after emergency TIPS for uncontrolled acute variceal bleeding in patients with cirrhosis and portal hypertension.

In this specific subset of patients, sepsis was the cause of mortality in $8 \%$ of the 73 patients included. Moreover, despite the fact that all patients received antibiotic prophylaxis (as part of the standard of care treatment of cirrhotic patients hospitalised for acute variceal bleeding), $42 \%$ of patients developed an in-hospital infection, including multi-drug resistant bacteria (23\%). The authors did not mention whether any of the patients who developed infection postTIPS placement had previously had an episode of infection prior to the procedure. ${ }^{26}$

While none of the aforementioned studies stated whether an episode of infection in the months prior to TIPS placement increased the risk of infection post-TIPS procedure, they all documented infection as a common and serious complication seen in patients who had TIPS.

The MELD-Na (and some of the tests used to calculate the MELD-Na) score was higher in the group of patients who had an infection before the TIPS. This indicates that the group of patients who had an episode of bacterial or fungal infection before TIPS had more advanced liver disease. Therefore, the fact that the MELD-Na score in this group remained higher after the TIPS placement was not surprising. Similarly, the fact that there was a higher percentage of patients in Group A who had PSE prior to TIPS was also expected, given the fact that infection is one of the wellknown triggers of PSE. ${ }^{27}$

Whether the higher MELD-Na or the previous infection determined the higher number of infections post-TIPS cannot be entirely determined because patients with a higher MELD score, by 
definition, have more advanced stages of the liver disease, and are therefore more likely to develop any of the complications of cirrhosis, including infection. However, this fact does not weaken the value of the results; in fact, it is likely that both a high MELD score and a history of infection in the 6 months prior to TIPS increase the risk of infection after the procedure. An episode of PSE pre-TIPS as a predictor of post-TIPS PSE has also been previously described..$^{14,25}$

Conversely to what was hypothesised by the authors, despite the higher frequency of postTIPS infection and post-TIPS PSE in Group A, the frequency of other complications of TIPS procedure, particularly hospitalisation, the need for TIPS revision, the need for liver transplant, or death, was not affected. Since this was a retrospective chart review study, this finding cannot be attributed to any specific measure intentionally taken. However, these results may be used as an alert. When deciding on TIPS placement in a patient who had an infection in the 6 months preceding the procedure, closer attention must be paid to early signs and symptoms of infection post-TIPS.

This study has some limitations: it lacks detailed information about which types of infection the patients had, the duration of the episodes, whether or not there were any infections by multidrug resistant organisms, and the exact time from infection to TIPS placement. In addition, information in regard to hospitalisations and other comorbidities that could have increased the risk of infection is also missing.

Despite these limitations, these results offer valuable information for the clinician treating patients with portal hypertension. The finding that an episode of infection in the 6 months preceding TIPS is related to post-TIPS infection, to the authors' knowledge, has not been previously described. This observation brings important considerations when treating this patient population. The first is the possibility of, whenever feasible, delaying the TIPS placement until possible infections have been ruled-out, and when present, treated. Secondly, while use of antibiotics must always be judiciously evaluated, further studies are warranted to consider the possible benefit of antibiotic prophylaxis to prevent post-TIPS infection in patients with a history of infection in the 6 months preceding TIPS placement, particularly those with a higher MELD-Na score. In addition, given the fact that an episode of infection prior to TIPS increases the risk of both PSE and infection post-TIPS, these results also highlight the importance of educating patients and care givers about the further increase in the risk of PSE post-TIPS in those with a history of infection prior to TIPS placement.

Finally, while no episodes of infection of the TIPS shunt itself (tipsitis) were diagnosed during the study period, pre-TIPS infection is a possible risk factor of tipsitis. This is particularly important to consider in those patients who are not candidates for liver transplant because treatment options for tipsitis are limited to orthotopic liver transplantation or lifetime antibiotics, as an infected TIPS shunt cannot be removed. ${ }^{5,15}$

\section{CONCLUSION}

Comparing patients with an episode of infection in the 6 months prior to TIPS placement (Group A) and those with no infection in the 6 months preceding TIPS (Group B), a statistically significant increased frequency of post-TIPS infection in patients in Group A compared to Group B was found (26.4\% versus $16.2 \%$ ). Using regression analysis, pre-TIPS infection was a predictor of post-TIPS infection ( $p=0.047$; OR: 2.08 ). It was also discovered that there was an increased frequency of post-TIPS PSE in Group A versus Group B. In Group A, 54.7\% ( $n=29)$ of the patients had an episode of PSE post-TIPS versus $39.6 \%(n=134)$ in Group B ( $p=0.046$; OR: 1.93). This finding was not surprising, given the well-known fact that infection is a trigger of PSE. However, it underscores the importance of educating patients and care givers about the further increased risk of PSE after TIPS placement. While these findings did not correlate with the frequency of other complications of TIPS procedure, namely hospitalisation within 90 days, acute kidney injury requiring haemodialysis, procedural haemorrhage, gastrointestinal haemorrhage, respiratory complications, or death, these results serve as an alert to the possibility of other complications of TIPS in this specific population. In addition, the results pave the path for further studies to determine the potential benefit of antibiotic prophylaxis in patients who had an episode of infection in the 6 months preceding TIPS placement. 


\section{References}

1. Boyer T et al. The role of transjugular intrahepatic portosystemic shunt (TIPS) in the management of portal hypertension: update 2009. Hepatology. 2010;51(1):306.

2. Colombato $L$. The role of transjugular intrahepatic portosystemic shunt (TIPS) in the management of portal hypertension. J Clin Gastroenterol. 2007;41(Suppl 3):S344-51.

3. Copelan A et al. Transjugular intrahepatic portosystemic shunt: indications, contraindications, and patient work-up. Semin Intervent Radiol. 2014;31(3):235-42.

4. Smith M, Durham J. Evolving Indications for Tips. Tech Vasc Interv Radiol. 2016;19(1):36-41.

5. Suhocki $P$ et al. Transjugular intrahepatic portosystemic shunt complications: prevention and management. Semin Intervent Radiol. 2015;32(2):123-32.

6. Kraglund $\mathrm{F}$ et al. Long-term effects and complications of the transjugular intrahepatic portosystemic shunt: a single-centre experience. Scand J Gastroenterol. 2019;54(7):899-904.

7. Lee E et al. Nationwide trends and predictors of inpatient mortality in 83884 transjugular intrahepatic portosystemic shunt. World J Gastroenterol. 2016;22(25):5780-9.

8. Casadaban L et al. MELD score for prediction of survival after emergent TIPS for acute variceal hemorrhage: derivation and validation in a 101-patient cohort. Ann Hepatol. 2015;14(3):380-8.

9. Ferral $\mathrm{H}$ et al. Survival after elective transjugular intrahepatic portosystemic shunt creation: prediction with model for endstage liver disease score. Radiology. 2004;231(1):231-6.

10. Hsu CY et al. Comparison of the model for end-stage liver disease (MELD), MELD-Na and MELDNa for outcome prediction in patients with acute decompensated hepatitis. Dig Liver Dis. 2010;42(2):137-42.

11. Ronald $\mathrm{J}$ et al. Albumin-bilirubin grade versus MELD score for predicting survival after transjugular intrahepatic portosystemic shunt (TIPS) creation. Diagn Interv Imaging. 2018;99(3):163-8

12. Roth $\mathrm{J}$ et al. MELD score as a predictor of mortality, length of hospital stay, and disease burden: a single-center retrospective study in 39,323 inpatients. Medicine (Baltimore). 2017;96(24):e7155

13. Schepke $M$ et al. Comparison of MELD, Child-Pugh, and Emory model for the prediction of survival in patients undergoing trans- jugular intrahepatic portosystemic shunting. Am J Gastroenterol. 2003;98(5):116774.

14. Bai M et al. Predictors of hepatic encephalopathy after transjugular intrahepatic portosystemic shunt in cirrhotic patients: a systematic review. J Gastroenterol Hepatol. 2011;26(6):943-51.

15. Kochar $\mathrm{N}$ et al. Tipsitis: Incidence and outcome-a single centre experience. Eur J Gastroenterol Hepatol. 2010;22(6):729-35.

16. Such J, Runyon B. Spontaneous bacterial peritonitis. Clin Infect Dis. 1998;27(4):669-74

17. Bunchorntavakul $\mathrm{C}$ et al. Bacteria infections in cirrhosis: a critical review and practical guidance. 2016;8(6):307-21.

18. Jalan $\mathrm{R}$ et al. Bacterial infections in cirrhosis: A position statement based on the EASL special conference 2013. J Hepatol. 2014;60(6):1310-24.

19. Fernández J, Gustot T. Management of bacterial infections in cirrhosis. J Hepatol. 2012;56(Suppl 1):S1-12.

20. Gustot T et al. Severe sepsis in cirrhosis. Hepatology. 2009;50(6):2022-33.

21. Schwabl P et al. Risk factors for development of spontaneous bacterial peritonitis and subsequent mortality in cirrhotic patients with ascites. Liver Int. 2015;35(9):2121-8.

22. Saad W. The history and future of transjugular intrahepatic portosystemic shunt: food for thought. Semin Intervent Radiol. 2014;31(3):258-61.

23. Centers for Disease Control and Prevention (CDC). National Health Interview Survey. Tables of Summary Health Statistics. 2019. Available at: http://www.cdc.gov/nchs/nhis/SHS/ tables.htm. Last accessed: 25 October 2019.

24. Deng $P$ et al. Risk factors contributing to early infection following transjugular intrahepatic portosystemic shunt in perioperative period. Ann Hepatol. 2016;15(5):752-6.

25. Vozzo C et al. Hospital readmission following transjugular intrahepatic portosystemic shunt: a 14-year singlecenter experience. Gastroenterol Rep (Oxf). 2019;goz063.

26. Allaire $M$ et al. SAT-O07-Survival and risk factors of mortality after emergency transjugular intrahepatic portosystemic shunt for uncontrolled acute variceal bleeding caused by cirrhosis-related portal hypertension. J Hepatol. 2019;70(1):e628.

27. Vilstrup $\mathrm{H}$ et al. Hepatic encephalopathy in chronic liver disease: 2014 practice guideline by the American association for the study of liver diseases and the European association for the study of the liver. Hepatology. 2014;60(2):71535. 